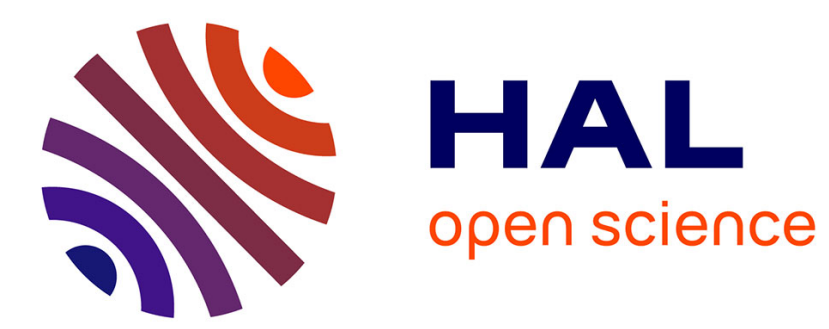

\title{
Fluage en compression d'un smectique B
}

P. Oswald

\section{- To cite this version:}

P. Oswald. Fluage en compression d'un smectique B. Journal de Physique Lettres, 1984, 45 (21), pp.1037-1044. 10.1051/jphyslet:0198400450210103700 . jpa-00232447

\section{HAL Id: jpa-00232447 https://hal.science/jpa-00232447}

Submitted on 1 Jan 1984

HAL is a multi-disciplinary open access archive for the deposit and dissemination of scientific research documents, whether they are published or not. The documents may come from teaching and research institutions in France or abroad, or from public or private research centers.
L'archive ouverte pluridisciplinaire HAL, est destinée au dépôt et à la diffusion de documents scientifiques de niveau recherche, publiés ou non, émanant des établissements d'enseignement et de recherche français ou étrangers, des laboratoires publics ou privés. 
Classification

Physics Abstracts

$61.30-62.20 \mathrm{H}$

\title{
Fluage en compression d'un smectique B
}

\author{
P. Oswald \\ Laboratoire de Physique des Solides, Bât. 510. \\ Université Paris-Sud, 91405 Orsay Cedex, France
}

(Reçu le 3 juillet 1984, accepté le 13 septembre 1984)

\begin{abstract}
Résumé. - Nous décrivons deux régimes de fluage observés en compression normale aux couches dans la phase $\mathrm{B}$ du 40.8 (butyloxybenzilidène octylaniline) : ils se caractérisent tous deux par une dépendance linéaire en contrainte de la vitesse de déformation et par une même énergie d'activation apparente. Un mécanisme lacunaire est proposé. A faible contrainte, les joints de grain du polycristal jouent le rôle de sources et de puits de lacunes et le fluage est gouverné par la diffusion intragranulaire des lacunes (fluage Nabarro-Herring). A plus forte contrainte, ce rôle est joué également par les dislocations coin qui participent à la déformation par montée pure (fluage du type Harper-Dorn). Ces expériences confirment la structure cristalline de la phase B.
\end{abstract}

\begin{abstract}
We describe two regimes of creep observed under a compression normal to the layers in the B phase of $\mathbf{4 0 . 8 ~ ( b u t y l o x y b e n z i l i d e n e ~ o c t y l a n i l i n e ) ~ : ~ b o t h ~ a r e ~ c h a r a c t e r i z e d ~ b y ~ a ~ l i n e a r ~ d e p e n - ~}$ dence of the strain rate versus stress and by the same apparent activation energy. A vacancy mechanism is proposed. For weak stresses, the grain boundaries of the polycrystal act as vacancy sources and sinks; the creep is then governed by the intragranular diffusion of the vacancy (Nabarro-Herring creep). For greater stresses, edge dislocations also act as vacancy sources or sinks and participate in the strain by pure climb (Harper-Dorn type creep). These experiments confirm the crystalline structure of the B phase.
\end{abstract}

\section{Introduction.}

La structure des phases smectiques $\mathbf{B}$ a fait l'objet ces dernières années de nombreux travaux théoriques et expérimentaux. Rappelons qu'il s'agit de phases lamellaires avec un ordre hexagonal dans le plan des couches. Différents modèles théoriques ont été proposés. Parmi eux celui de B. J. Birgeneau et J. D. Litster [1] alliant un ordre de position à courte distance dans les couches à un ordre à longue distance pour les orientations des liaisons des molécules proches voisines. Néanmoins les premières études aux rayons $\mathrm{X}$ sur la phase $\mathrm{B}$ du 40.8 (butyloxybenzilidène octylaniline) $[2,3]$ se révélèrent compatibles avec un ordre tridimensionnel. Des analyses récentes aux rayons $X$ sur films libres [4] et à haute résolution sur échantillons massifs [5] permirent de préciser les longueurs de cohérence mises en jeu et de montrer définitivement qu'il s'agissait d'un cristal tridimensionnel. Ce résultat a été confirmé par des mesures mécaniques à basse fréquence du module de cisaillement dans le plan des couches [6, 7] et entre les couches [8-10] ainsi que par atténuation ultra-sonore [11]. 
Néanmoins la phase $\mathrm{B}$ du 40.8 présente certaines propriétés qui la diffèrent des cristaux ordinaires, à savoir de fortes fluctuations du directeur $\left(\sim 4^{\circ}[2]\right)$, une rotation uniaxiale des molécules autour de leur axe, et surtout, un très faible couplage énergétique entre couches voisines. En sont pour preuves la petitesse devant les autres modules élastiques $\left(\sim 5 \times 10^{8} \mathrm{dynes} / \mathrm{cm}^{2}\right)$ du module de cisaillement parallèle aux couches $\left(\sim 10^{6}\right.$ dynes $\left./ \mathrm{cm}^{2}\right)$, ou encore les anomalies observées aux rayons X (forte diffusion diffuse [5]). Notons enfin l'existence dans ces systèmes d'une instabilité d'ondulation des couches [12] encore mal comprise et très peu étudiée, mais présentant des analogies avec celle bien connue des smectiques A [13].

Cet ensemble de propriétés inhabituelles pour un cristal, cette ressemblance de comportement sous dilatation avec le smectique $\mathrm{A}$, et l'absence quasi totale dans la littérature de données expérimentales sur les mécanismes de déformation des cristaux moléculaires, nous ont conduits à reprendre des expériences de fluage en compression normale aux couches analogues à celles que nous avions déjà réalisées en smectique $\mathrm{A}$ [14].

Après quelques rappels sur l'appareillage et le mode opératoire, nous décrivons les résultats expérimentaux. Il apparaît, comme attendu, que le smectique B est beaucoup plus proche des solides ordinaires que du smectique A. Aussi, est-ce dans le cadre de la plasticité des solides cristallins [18] que nous analysons les différents régimes de fluage observés. En conclusion, nous suggérons quelques expériences nouvelles devant permettre une meilleure compréhension des mécanismes mis en jeu au cours de la déformation.

\section{Appareillage et mode opératoire.}

La machine de fluage utilisée a déjà été décrite dans un article antérieur [14]. L'échantillon est placé entre deux lames de verre parallèles (à mieux que $10^{-3} \mathrm{rd}$ ) traitées au silane afin d'assurer un bon ancrage homéotrope. La qualité des échantillons est contrôlée par observation directe au microscope polarisant. Ils sont préparés par refroidissement $\left(1^{\circ} \mathrm{C} / \mathrm{min}\right)$ à partir de la phase nématique. Rappelons que le 40.8 présente les transitions de phases suivantes :

$$
\text { cristal } \stackrel{33^{\circ} \mathrm{C}}{\Longleftrightarrow} \mathrm{Sm} \mathrm{B} \stackrel{49^{\circ} \mathrm{c}}{\Longleftrightarrow} \mathrm{Sm} \mathrm{A} \stackrel{64 \mathrm{c}}{\Longleftrightarrow} \text { Nématique . }
$$

Les charges appliquées vont de $1 \mathrm{~g}$ à $200 \mathrm{~g}$. La surface des échantillons peut varier de $0,2 \mathrm{~cm}^{2}$ à $2 \mathrm{~cm}^{2}$ pour une épaisseur comprise entre $100 \mu \mathrm{m}$ et $1 \mathrm{~mm}$. L'amplitude totale du fluage n'excède jamais $15 \%$ de l'épaisseur initiale de l'échantillon. Après chaque essai, l'échantillon est ramené à son épaisseur initiale puis préparé à nouveau après passage en phase nématique. Cette procédure permet une bonne reproductibilité des résultats.

\section{Résultats expérimentaux.}

Tous nos essais ont été réalisés en compression normale aux couches. L'expérience montre que, quelle que soit la contrainte appliquée (toujours inférieure à $4 \times 10^{5}$ dynes $/ \mathrm{cm}^{2}$ ), la vitesse de fluage $\dot{\varepsilon}$ reste constante au cours du temps. Aucun régime transitoire visible ne précède le régime stationnaire. Nous avons reporté sur la figure 1 la vitesse de fluage pour des charges croissantes à diverses températures. Dans cet exemple l'épaisseur de l'échantillon est de $600 \mu \mathrm{m}$, sa surface de $0,45 \mathrm{~cm}^{2}$ et l'angle des lames voisin de $5 \times 10^{-4} \mathrm{rd}$. Pour cet échantillon la reproductibilité des résultats est assez bonne $( \pm 20 \%)$. Un recuit prolongé de l'échantillon ( 24 heures ou plus) avant déformation ne modifie pas de façon significative les vitesses de fluage mesurées. Des écarts plus importants $( \pm 50 \%)$ ont été relevés entre échantillons différents mais n'ont pu être reliés à un effet précis (vieillissement du produit, teneur en impuretés et en poussières, qualité du traitement au silane...). 


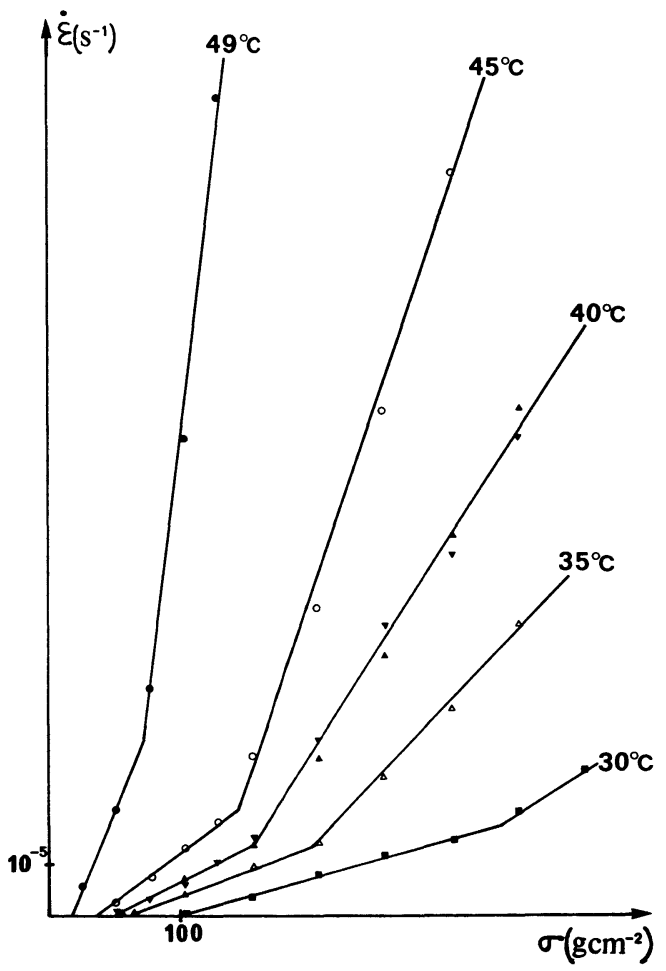

Fig. 1. - Vitesse de déformation en fonction de la contrainte à différentes températures $\left(S=0,4 \mathrm{~cm}^{2}\right.$, $d=600 \mu \mathrm{m}$ et $\alpha=5 \times 10^{-4} \mathrm{rd}$ ).

[Strain rate as a function of applied stress for different temperatures $\left(S=0.4 \mathrm{~cm}^{2}, d=600 \mu \mathrm{m}\right.$ and $\left.\alpha=5 \times 10^{-4} \mathrm{rd}\right)$.]

L'analyse des courbes reportées en figure 1 montre clairement que la vitesse de fluage peut être schématisée par la séquence simple suivante :

$$
\begin{array}{ll}
\sigma<\sigma_{1} & \dot{\varepsilon}=0 \\
\sigma_{1}<\sigma<\sigma_{2} & \dot{\varepsilon}=A_{1}\left(\sigma-\sigma_{1}\right) \\
\sigma>\sigma_{2} & \dot{\varepsilon}=A_{1}\left(\sigma-\sigma_{1}\right)+A_{2}\left(\sigma-\sigma_{2}\right)
\end{array}
$$

$\sigma$ représente la contrainte appliquée et $\dot{\varepsilon}$ la vitesse de déformation (exprimée en $\mathrm{s}^{-1}$ ). On vérifie aisément qu'une représentation par une loi puissance unique du type $\dot{\varepsilon}=\left(\sigma-\sigma_{1}\right)^{n}$, classique en métallurgie, ne peut rendre compte des résultats expérimentaux. Signalons enfin que pour la clarté de la figure 1, nous avons omis d'indiquer les points expérimentaux de fluage nul à $\sigma<\sigma_{1}$. Des essais systématiques sur des échantillons d'épaisseurs différentes $(200 \mu \mathrm{m}<d<900 \mu \mathrm{m})$ de surfaces variées $\left(0,5 \mathrm{~cm}^{2}<S<2 \mathrm{~cm}^{2}\right)$ et d'angles modérés $\left(\alpha<10^{-2} \mathrm{rd}\right)$ ont montré que les grandeurs $A_{1}, A_{2}, \sigma_{1}$ et $\sigma_{2}$ étaient indépendantes de ces variables géométriques. Néanmoins nous avons constaté une augmentation marquée de $A_{2}$ pour des angles très élevés $\left(\alpha \sim 3 \times 10^{-2} \mathrm{rd}\right)$ des lames de verre, les autres quantités restant sensiblement inchangées (Fig. 2). Le diagramme en température de la figure 3 montre que les grandeurs $A_{1}$ et $A_{2}$ sont thermiquement activées avec la même énergie d'activation $E \sim 0,7 \mathrm{eV}$. Cette énergie d'activation augmente à la transition Sm A-Sm B, ce qui peut traduire un effet prétransitionnel. Enfin on constate que $\sigma_{2} \sim 3 \sigma_{1}$ 


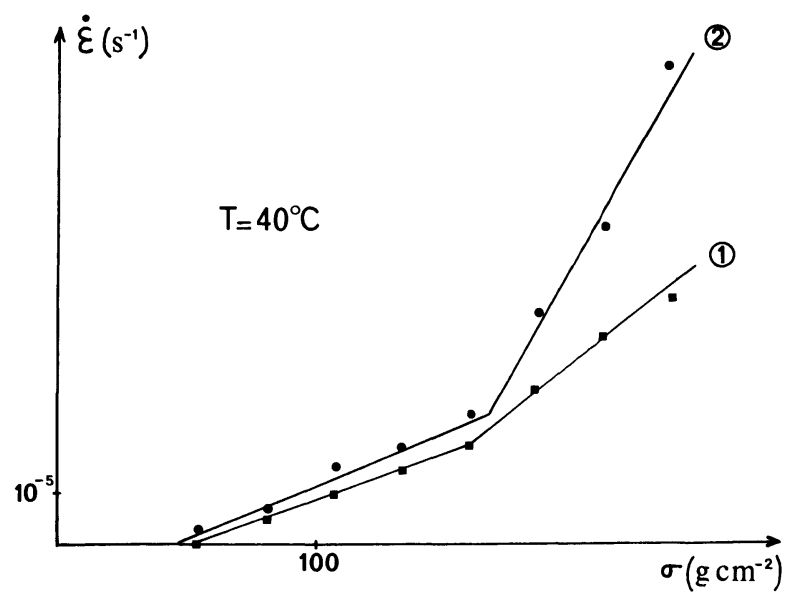

Fig. 2. - Influence de l'angle $\alpha$ des lames limitant l'échantillon sur la vitesse de fluage $\left(S=0,75 \mathrm{~cm}^{2}\right.$, $d=900 \mu \mathrm{m}$, (1) $\alpha=10^{-3} \mathrm{rd}$, (2) $\alpha=3 \times 10^{-2} \mathrm{rd}$ ).

[Influence on the strain rate of the angle $\alpha$ of the slides limiting the sample $\left(S=0.75 \mathrm{~cm}^{2}, d=900 \mu \mathrm{m}\right.$, (1) $\alpha=10^{-3} \mathrm{rd}$, (2) $\alpha=3 \times 10^{-2} \mathrm{rd}$ ).]

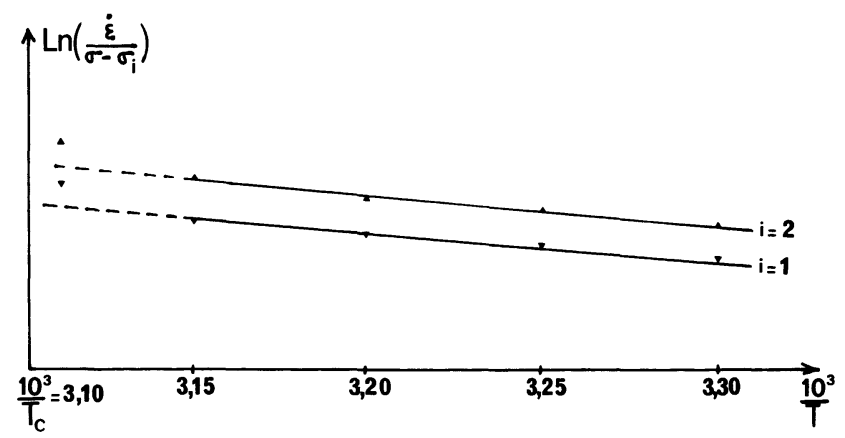

Fig. 3. - Diagramme d'Arrhenius pour les termes $\frac{\dot{\varepsilon}}{\sigma-\sigma_{i}}(i=1,2)$ relatifs aux deux régimes de fluage. On mesure la même énergie d'activation $E=0,67 \mathrm{eV}$ dans les deux cas.

[Arrhenius plot for the terms $\frac{\dot{\varepsilon}}{\sigma-\sigma_{i}}(i=1,2)$ corresponding to the two creep regimes. We measure the same activation energy, $E=0.67 \mathrm{eV}$, in both cases.]

à toute température. La figure 4 montre l'évolution linéaire de la limite d'élasticité $\sigma_{1}$ en fonction de la température. On remarquera qu'elle devient très petite à la transition $\mathrm{Sm} \mathrm{A} \Leftrightarrow \mathrm{Sm} \mathrm{B}$. Il convient de noter que cette contrainte seuil n'est pas d'origine élastique, les modules d'élasticité en cisaillement [10] ou en compression normale aux couches (Fig. 5) variant très peu avec la température, contrairement à $\sigma_{1}$.

\section{Discussion.}

Nous ne disposons actuellement d'aucune donnée expérimentale sur la microstructure du cristal avant ou après déformation. Cette absence d'information fait qu'il est toujours difficile, voir hasardeux, d'attribuer un mécanisme précis à un régime de fluage donné. Néanmoins quelques 


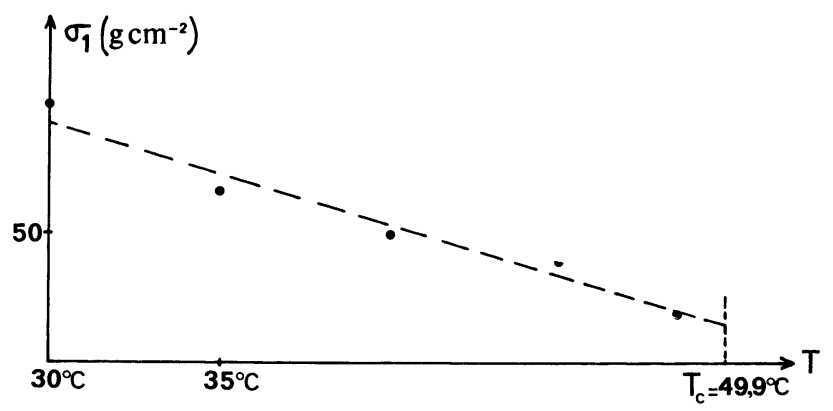

Fig. 4. - Contrainte d'écoulement $\sigma_{1}$ en fonction de la température.

[Flow stress $\sigma_{1}$ versus temperature.]

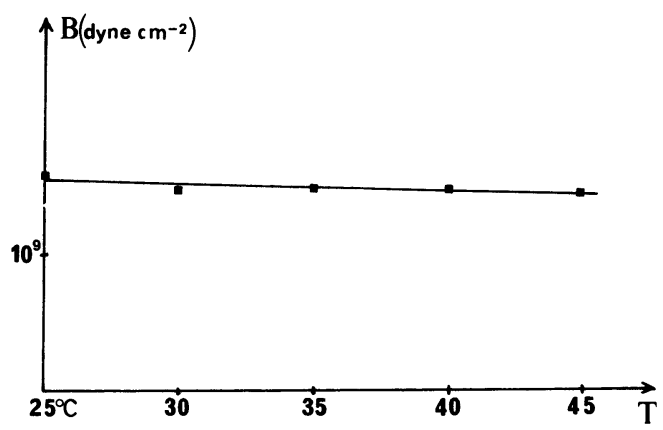

Fig. 5. - Dépendance en température du module d'élasticité $B$ en compression normale aux couches. Nous l'avons mesuré au moyen de l'appareillage décrit dans [28].

[Temperature dependence of the bulk modulus $B$ under a compression normal to the layers. Measurement made with the apparatus described in [28].]

remarques importantes permettent de situer le problème. La première concerne la dépendance linéaire en contrainte habituellement observée dans les métaux à haute température (proche du point de fusion) et pour de faibles contraintes. La seconde, importante, traduit l'égalité des énergies d'activation pour les deux régimes observés (Fig. 3) et suggère l'existence d'un mécanisme de base commun, par exemple la diffusion volumique de lacunes. Enfin l'augmentation marquée de la vitesse de fluage dans le second régime pour des angles élevés indique un mécanisme par montée de dislocations coin de vecteur de Burgers normal aux couches. Ces remarques nous amènent à proposer deux modèles de fluage classiques en métallurgie.

A faible contrainte, un fluage par diffusion intragranulaire $[15,16]$ peut rendre compte de nos mesures. Ce modèle suppose l'existence de grains d'orientations différentes du réseau hexagonal dans le plan des couches. Les observations aux rayons X de P. Pershan et al. [5] le confirment mais ne permettent pas de mesurer leurs dimensions caractéristiques. Par ailleurs, l'existence de ces grains a été avancée par A. J. Dianoux et al. [29] dans la phase Sm G du TBBA, de structure analogue à la phase $\mathrm{B}$, pour expliquer les écarts entre les coefficients d'autodiffusion mesurés sur poudre et sur monocristal. Dans la phase B, ces grains ne sont malheureusement pas visibles par microscopie optique, l'échantillon restant globalement uniaxe. Néanmoins, les germes de croissance sont visibles à la transition $\mathrm{Sm} \mathrm{A-Sm} \mathrm{B}$. Leur aspect ressemble à celui de domaines de 
croissance à contours digités des systèmes colonnaires hexagonaux de molécules discoïdes [17] et leur nombre par unité de surface indique que la taille des grains n'excède pas $100 \mu \mathrm{m}$. Un calcul classique, fondé sur l'existence d'un gradient de concentration de lacunes entre les faces du grain en tension et en compression conduit à la loi de fluage suivante [15] :

$$
\dot{\varepsilon}=C \frac{D \Omega}{k T d^{2}}\left(\sigma-\sigma_{1}\right)
$$

$d$ est la dimension caractéristique d'un grain (de l'ordre de $50 \mu \mathrm{m}$ ) $\Omega$ le volume atomique $\left(7,5 \times 10^{-22} \mathrm{~cm}^{3}\right), D$ le coefficient d'autodiffusion et $C$ une constante numérique qui dépend de la forme du grain, mais toujours voisine de 10 [18]. La contrainte seuil $\sigma_{1}$ que l'on observe quelquefois dans ce type de fluage traduit que les joints de grain n'agissent pas comme des sources ou des puits parfaits de lacunes. Dans ces conditions une différence de potentiel chimique $\Delta \mu$ minimale entre le joint et l'intérieur du grain est nécessaire pour provoquer l'émission ou l'absorption d'une lacune [19]. Il en résulte la contrainte seuil

$$
\sigma_{1}=\frac{\Delta \mu}{\Omega}
$$

nécessaire pour initier le fluage, d'où un écoulement du type Bingham. Ce modèle attribue l'énergie d'activation mesurée $(0,67 \mathrm{eV})$ au coefficient d'autodiffusion en volume inconnu à notre connaissance. Pour l'évaluer prenons $d=50 \mu \mathrm{m}$ et $C=10$. A $40^{\circ} \mathrm{C}$ nous mesurons $\dot{\varepsilon}=1,3 \times 10^{-5} \mathrm{~s}^{-1}$ pour $\sigma-\sigma_{1}=2,6 \times 10^{4}$ dynes $/ \mathrm{cm}^{2}$. La formule (2) conduit à $D \sim 7 \times 10^{-8} \mathrm{~cm}^{2} / \mathrm{s}$. Des mesures par RMN dans la phase smectique A du même composé donnent $D \sim 2 \times 10^{-7} \mathrm{~cm}^{2} / \mathrm{s}$ à $50^{\circ} \mathrm{C}$ [20]. Notre valeur, 3 fois plus petite, est donc raisonnable, sachant qu'en moyenne le coefficient de diffusion varie d'un facteur 5 à la transition $\mathrm{Sm} \mathrm{A} \Leftrightarrow \mathrm{Sm} \mathrm{B}$ dans d'autres composés connus [21].

A plus fortes contraintes $\left(\sigma>\sigma_{2} \sim 3 \sigma_{1}\right)$ se superpose au régime précédent un processus de déformation que nous attribuons à la montée de dislocations coin situées dans les couches et de vecteur de Burgers suivant l'axe c (normal aux couches). Supposons qu'elles soient réparties de façon uniforme et désignons par $\rho$ leur densité supposée constante au cours de la déformation. Cette situation est peu fréquente dans les métaux mais se rencontre quelquefois à très haute température et faible contrainte (fluage de Harper-Dorn [23]). Si la distance moyenne entre crans est petite devant la distance séparant deux dislocations, la montée est contrôlée par la diffusion en volume [24]. Nous ferons cette hypothèse par la suite. J. Friedel montre que le taux de déformation s'écrit dans ces conditions [22] :

$$
\dot{\varepsilon}=\frac{\rho \Omega}{\operatorname{Ln}\left(1 / \rho^{1 / 2} b\right)} \frac{D}{k T}\left(\sigma-\sigma_{2}\right) .
$$

Nous avons introduit de façon phénoménologique la contrainte seuil $\sigma_{2}$ dans cette formule. Elle exprime, comme dans le cas des joints de grain, que les dislocations n'agissent pas comme des sources ou des puits parfaits de lacunes et qu'il faut une différence de potentiel chimique au moins égale à $3 \Delta \mu$ pour que montent les dislocations. Dans ce modèle l'énergie d'activation est celle du coefficient d'autodiffusion comme dans le cas précédent. Ce point est conforme à l'expérience. Pour évaluer la densité $\rho$ remarquons qu'une variation d'angle $\Delta \alpha \sim 3 \times 10^{-2}$ rd double la vitesse de fluage d'un échantillon d'épaisseur $d=600 \mu \mathrm{m}$. Une évaluation grossière conduit à $\rho \sim \frac{\Delta \alpha}{b d} \sim 10^{6} \mathrm{~cm}^{-2}$ pour un échantillon à lames à faces parallèles $\left(\alpha<10^{-3} \mathrm{rd}\right)$. Considérant cette valeur et celle trouvée précédemment pour le coefficient d'autodiffusion à $40^{\circ} \mathrm{C}$, nous 
déduisons à partir de (4) $\dot{\varepsilon}_{\mathrm{th}}=1,6 \times 10^{-5} \mathrm{~s}^{-1}$ pour $\sigma-\sigma_{2}=10^{5}$ dynes $/ \mathrm{cm}^{2}$. La valeur expérimentale $\dot{\varepsilon}_{\text {exp }}=2,6 \times 10^{-5} \mathrm{~s}^{-1}$ est proche. Ce bon accord rend plausible les deux mécanismes proposés.

\section{Conclusion.}

Ces quelques expériences montrent clairement que le smectique B est beaucoup plus proche des solides ordinaires que des cristaux liquides smectiques A sur le plan rhéologique. Ce point est donc bien conforme aux analyses structurales récentes. Néanmoins de nombreuses questions restent posées, en particulier l'origine exacte du seuil d'élasticité $\sigma_{1}$ et sa forte dépendance en température. D'autre part il apparaît nécessaire, pour tester les modèles proposés, de développer des méthodes microscopiques qui permettent d'accéder à la microstructure du cristal (imagerie $\mathbf{X}$ ou microscopie électronique par observation de répliques de surfaces de fracture); une mesure indépendante (par RMN ou par diffusion quasi-élastique de neutrons [21]) du coefficient d'autodiffusion et de son énergie d'activation s'avère, elle aussi, indispensable. Notons que ces modèles reposent sur la diffusion volumique des lacunes dont nous avons supposé l'existence implicitement. Leur mise en évidence directe, par annihilation de positons par exemple [25], serait particulièrement intéressante et montrerait de façon définitive qu'il s'agit d'un vrai cristal. D'autre part, une étude théorique soignée des dislocations tenant compte de la très forte anisotropie des modules d'élasticité, devrait permettre une meilleure compréhension des mécanismes mis en jeu. Enfin l'existence de nombreuses fautes d'empilement [26] suggère la possibilité de polytypisme [27] dans ces matériaux dont nous nous proposons d'étudier les conséquences rhéologiques dans l'avenir. Une expérience de fluage en cisaillement parallèle aux couches devrait apporter quelques renseignements à ce sujet.

\section{Remerciements.}

Je remercie M. Kléman qui m'a conseillé tout au long de cette étude, ainsi que Y. Quéré, J. P. Poirier et A. M. Levelut qui m'ont fait part de leurs commentaires. Je remercie également P. Germain pour la synthèse du 40.8 et $\mathrm{V}$. Klein pour son assistance technique.

\section{Bibliographie}

[1] Birgeneau, R. J., Litster, J. D., J. Physique Lett. 39 (1978) L-399.

[2] Levelut, A. M., Doucet, J., Lambert, M., J. Physique 35 (1974) 773.

[3] Doucet, J., Levelut, A. M., J. Physique 38 (1977) 1163.

[4] Moncton, D. E., Pindak, R., Phys. Rev. Lett. 43 (1979) 701.

[5] Pershan, P., Zeppli, G., Litster, J. D., Birgeneau, R., Mol. Cryst. Liq. Cryst. 67 (1981) 205.

[6] Pindak, R., Bishop, D. J., Sprenger, W. O., Phys. Rev. Lett. 44 (1980) 1461.

[7] Tarczon, J. C., Miyano, K., Phys. Rev. Lett. 46 (1981) 119.

[8] Cagnon, M., Durand, G., Phys. Rev. Lett. 45 (1980) 1418.

[9] Cagnon, M., Durand, G., J. Physique Lett. 42 (1981) L-451.

[10] Cagnon, M., Palierne, J. F., Durand, G., Mol. Cryst. Liq. Cryst. Lett. 82 (1982) 185.

[11] Thiriet, Y., Martinoty, P., J. Physique Lett. 43 (1982) L-137.

[12] Ribotta, R., Phys. Lett. A 56 (1976) 130.

[13] Ribotta, R., Durand, G., J. Physique 38 (1977) 179.

[14] Oswald, P., C.R. Séan. Acad. Sci., Paris Sér. II 296 (1983) 1385.

[15] Nabarro, F. R. N., Philos. Mag. 16 (1967) 231.

[16] Herring, C., J. Appl. Phys. 21 (1950) 437.

[17] Billard, J., Dubois, J. C., Nguyen HuU Tinh, Zann, A., Nouv. J. Chim. 2 (1978) 535. 
[18] PoIRIER, J. P., Plasticité à haute température des solides cristallins (Ed. Eyrolles) 1976.

[19] Ashby, M. F., Scr. Metall. 3 (1969) 837.

[20] Blinc, R., Pirs, J., Zupancic, I., Phys. Rev. Lett. 30 (1973) 546.

[21] KRÜGER, G. J., Phys. Rep. 82 (1982) 231.

[22] Friedel, J., Dislocations (Pergamon Press) 1984.

[23] Langdon, T. G., Yavari, P., Acta Metall. 30 (1982) 881.

[24] Edelin, G., Philos. Mag. 23 (1971) 1547.

[25] QUÉRÉ, Y., Communication personnelle.

[26] Levelut, A. M., Communication personnelle.

[27] PoIRIER. J. P.. Communication personnelle.

[28] Oswald, P., Lefur, D., C.R. Hebd. Séan. Acad. Sci., Paris Sér. II 297 (1983) 699.

[29] Dianoux, A. J., Volino, F., Heidemann, A., Hervet, H., J. Physique Lett. 36 (1975) L-275. 